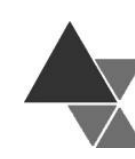

\title{
O auxílio alimentação no Brasil: seu efeito na distribuição da renda e no Índice de Massa Corporal dos empregados, conforme dados da Pesquisa de
}

Orçamentos Familiares de 2008-2009

\author{
Rodolfo Hoffmann¹ e Letícia Alves Tadeu Santiago ${ }^{2}$
}

Lei de 1976 criou o Programa de Alimentação do Trabalhador (PAT), dando incentivo fiscal às empresas que promovessem a alimentação de seus empregados. A regulamentação estabelece que isso pode ser feito mantendo serviço próprio de refeições ou, indiretamente, facilitando sua aquisição por meio de tíquetes ou cartões específicos. A Pesquisa de Orçamentos Familiares de 2008-2009 registrou o valor do Auxílio Alimentação como parte da remuneração dos empregados. Esses dados são utilizados nesta pesquisa para analisar como esse auxílio afeta a distribuição da renda no Brasil e também o efeito do auxílio alimentação sobre características antropométricas dos beneficiados. Embora a lei que criou o auxílio considerasse que deveriam ser atendidos prioritariamente os trabalhadores de baixa renda, verifica-se que esse auxílio é um componente levemente regressivo da renda familiar per capita, beneficiando mais os trabalhadores relativamente bem remunerados. Em relação às características antropométricas, controlando o efeito da renda, não foi possível detectar qualquer impacto do auxílio alimentação no Índice de Massa Corporal (IMC) ou na probabilidade de sobrepeso ou obesidade em homens. Mas há evidências de que, em algumas circunstâncias, o auxílio favorece a obesidade de mulheres.

Palavras-chave: auxílio alimentação, Programa de Alimentação do Trabalhador, distribuição da renda, IMC, obesidade.

\section{How a tax incentive to provide meals to workers in Brazil affects income distribution and the Body Mass Index (BMI) of employees}

A law of 1976 created the Brazilian Workers' Feeding Program, giving companies a tax incentive to improve nutrition of their employees. Its regulation establishes that employers may provide onsite meals to their employees or, indirectly, facilitate its acquisition by means of specific tickets or cards. The 20082009 Family Budget Survey registered the value of such food aid as part of the employees' salary. Here

${ }^{1}$ Professor Sênior da ESALQ-USP, com apoio do CNPq. E-mail: hoffmannr@usp.br

2 Doutoranda em Economia Aplicada da ESALQ-USP. E-mail: leticiaalvests@yahoo.com.br

Os autores agradecem a Angela Kageyama e Josimar Gonçalves de Jesus pelas sugestões e correções em versão preliminar do artigo. 
the Survey's data are used to analyze how that food aid affects the per capita family income distribution in Brazil. Although the law stated that low-earnings workers should be given priority, the data reveal that this specific food aid is a slightly regressive component of the per capita family income, as it benefits more the better paid workers. We also analyzed the effect of this aid on employees' anthropometric characteristics. Controlling for the effect of income, there is no evidence that this food had any impact on the BMI or on the probability of overweight or obesity in men. However, the data indicate that, in some circumstances, that aid promotes obesity of women.

Key-words: food aid, Brazilian workers' feeding program, income distribution, BMI, obesity.

\section{INTRODUÇÃO}

Em 14 de abril de 1976 foi sancionada no Brasil a Lei $\mathrm{n}^{\mathrm{O}} 6.321^{3}$ criando o Programa de Alimentação do Trabalhador (PAT) ${ }^{4}$, que posteriormente foi regulamentado pelo Decreto $\mathrm{n}^{\mathrm{o}}$ 5, de 14 de janeiro de 1991. Desde então esse programa está ativo e completou 40 anos em 2016. O objetivo era melhorar as condições nutricionais dos trabalhadores de baixa renda ${ }^{5}$ ao proporcionar-lhes uma alimentação adequada e, dessa maneira, contribuir com a diminuição do número de casos de doenças relacionadas à alimentação e à nutrição ${ }^{[1]}$.

O PAT foi um programa que se expandiu rapidamente nos centros mais industrializados e passou a envolver o maior volume de recursos entre os componentes do Programa Nacional de Alimentação e Nutrição (Pronan) ${ }^{[2]}$. Ao longo dos anos muitas foram as alterações nos regulamentos e nas leis que regem o PAT; uma das mais importantes foi a constituição da Comissão Tripartite do PAT, instituída pela Portaria Interministerial 478, de 1 de novembro de $2005^{[3]}$. Compõem essa comissão membros representantes do governo, de entidades patronais e de entidades representantes de trabalhadores, que tem como finalidade acompanhar, avaliar e aperfeiçoar o PAT, dentre outras atribuições, possibilitando que o programa seja cada vez mais expressivo no cumprimento de seus objetivos e metas.

No ano de seu lançamento, o PAT atendeu, aproximadamente, 760 mil trabalhadores. Esses números se expandiram e em 1992 o programa chegou a atender 7,4 milhões de trabalhadores. Nesse mesmo ano o número de empresas participantes era de $39.181^{[2] .}$ A Tabela 1 mostra alguns dos principais números sobre a evolução do Programa de Auxílio ao Trabalhador, referentes aos meses de dezembro dos anos de 2008 a 2016.

3 "Dispõe sobre a dedução, do lucro tributável para fins de imposto sobre a renda das pessoas jurídicas, do dobro das despesas realizadas em programas de alimentação do trabalhador". Disponível em: http://www.planalto.gov.br/ccivil_03/leis/L6321.htm

${ }^{4}$ De acordo com Carvalho da Silva (1995)[2] o PAT foi iniciado pelo Ministério do Trabalho em 1977 e ofereceu refeições de 1.400 e 300 calorias a trabalhadores de baixa renda, a preços de até $20 \%$ do custo. $\mathrm{O}$ restante do custo era subsidiado pelo setor público e os empregadores.

${ }^{5}$ Para o PAT os trabalhadores de baixa renda são aqueles que recebem até cinco salários mínimos conforme estabelecido no Decreto no 5/1991. 
Tabela 1. Evolução dos principais dados relacionados ao Programa de Alimentação do Trabalhador, no período de 2008-2016

\begin{tabular}{cccccc}
\hline Ano & $\begin{array}{c}\text { Trabalhadores } \\
\text { Beneficiados } \\
(\mathrm{mil})\end{array}$ & $\begin{array}{c}\text { Empresas } \\
\text { Beneficiárias } \\
(\mathrm{mil})\end{array}$ & $\begin{array}{c}\text { Empresas } \\
\text { Fornecedoras } \\
\left(\text { mil }^{2}\right.\end{array}$ & $\begin{array}{c}\text { Empresas } \\
\text { Prestadoras }^{3}\end{array}$ & $\begin{array}{c}\text { Nutricionistas } \\
\text { (mil) }\end{array}$ \\
\hline 2008 & 13.425 & 103,1 & 4,4 & 110 & 7,3 \\
2009 & 14.343 & 118,1 & 5,7 & 131 & 9,4 \\
2010 & 15.365 & 134,2 & 6,9 & 152 & 11,7 \\
2011 & 16.248 & 152,3 & 8,2 & 173 & 14,0 \\
2012 & 17.585 & 168,9 & 9,4 & 194 & 16,2 \\
2013 & 18.330 & 185,7 & 10,9 & 215 & 20,3 \\
2014 & 19.006 & 204,7 & 12,2 & 232 & 22,2 \\
2015 & 19.559 & 222,9 & 13,4 & 248 & 23,9 \\
2016 & 20.102 & 239,9 & 14,3 & 269 & \\
\hline
\end{tabular}

Fonte: Elaborada pelos autores com base nos dados do Ministério do Trabalho e Emprego (MTE), Secretaria de Inspeção do Trabalho (SIT), Departamento de Segurança e Saúde no Trabalho (DSST), Coordenação do Programa de Alimentação do Trabalhador (COPAT) e Programa de Alimentação do Trabalhador (PAT) [4].

(1) Os dados são referentes ao mês de dezembro de cada ano. Dados de 2008 a 2015 foram acessados em 27/09/2016 e os de 2016 foram em $03 / 08 / 2017$.

(2) Empresa Fornecedora é aquela que prepara e comercializa a alimentação (refeição pronta ou cesta de alimentos) para outras empresas.

(3) Empresa Prestadora é a responsável por administrar documentos de legitimação, sejam impressos ou na forma de cartões eletrônicos/magnéticos, para aquisição de gêneros alimentícios em supermercados (alimentação convênio) ou para refeições em restaurantes (refeição-convênio) (DIEESE, 2013, p.55) [1].

Pode-se observar que entre dezembro de 2008 e dezembro de 2016 o número de trabalhadores beneficiados pelo programa aumentou 50\%. Já o número de empresas beneficiárias, ou seja, aquelas que concedem o benefício a seus trabalhadores, cresceu $133 \%$. Quanto ao número de profissionais de nutrição, o aumento no mesmo período foi de $226 \%$. No mês de dezembro de 2016, o programa atendeu cerca de 20 milhões de trabalhadores. Desse total, cerca de 3,1 milhões recebem acima de cinco salários mínimos e 16,9 milhões recebem até cinco salários mínimos. Observa-se, portanto, que o PAT também favorece trabalhadores que não fazem parte do grupo prioritário ${ }^{6}$.

Livro comemorativo dos 40 anos do PAT organizado por Mazzon ${ }^{[3]}$ é muito rico em informações sobre a evolução do programa, usando essencialmente dados do MTE. Assinala, por exemplo, que "O início do programa foi marcado pela adesão de grandes empresas; entretanto, com o passar do tempo a participação de empresas pequenas e médias aumentou e tornou-se uma realidade. Em 1977, o número

${ }^{6}$ As empresas que participam do PAT não são obrigadas a oferecer alimentação a todos os seus empregados, devendo dar prioridade àqueles que recebem até 5 salários mínimos. médio de trabalhadores beneficiados por empresa era de 597, enquanto em 2015 esse valor foi de 87,4 " (p. 144). Infelizmente o livro tende a ser laudatório do programa. Nas conclusões afirma que o PAT é "o mais longevo programa socioeconômico no Brasil” (p. 257), esquecendo que o Programa Nacional de Alimentação Escolar (Merenda Escolar) foi criado em 1955 e é o mais antigo programa público de segurança alimentar e nutricional do Brasil. Ressalta-se, nesse livro, que "Em relação ao perfil de renda dos trabalhadores beneficiados, há vinte anos (1996), cerca de 60,3\% recebiam até cinco salários mínimos; esse indicador aumentou para 74,7\% em 2006 e alcançou $83,2 \%$ no final de 2015 ” (p. 257-258), sem perceber que mesmo essa última porcentagem não mostra que o programa seja focalizado nos relativamente pobres. Veremos, adiante, que os dados da POF 2008-2009 indicam que o auxílio alimentação é até mesmo ligeiramente regressivo. 
Mesmo o PAT sendo um programa federal de auxilio alimentação envolvendo milhões de trabalhadores e um enorme volume de recursos, na revisão da literatura foi encontrado apenas um trabalho publicado usando os dados da POF 20082009 para analisar o acesso dos trabalhadores aos benefícios alimentação e refeição no Brasil: o artigo de Canella, Martins e Bandoni (2016) ${ }^{[5]}$. Os autores esclarecem que limitaram sua análise às pessoas ocupadas, "sendo elegíveis aqueles que se declararam: empregado privado, empregado público, empregado doméstico ou empregado temporário na área rural” (p. 3). Trata-se de todas as categorias referentes a empregados na classificação do trabalhador conforme sua posição na ocupação. Ao iniciar a apresentação dos resultados os autores afirmam que

\footnotetext{
"Ao todo a POF 2008-2009 estudou 190.159 indivíduos de todas as idades, dos quais 101.053 declararam alguma ocupação e 62.275 eram elegíveis para o estudo. Desses, 2.008 indivíduos (3,2\% da população ocupada) referiram receber benefícios alimentação e refeição"'[5] (p. 3).
}

Há, realmente, 190.159 pessoas de todas as idades na amostra da POF 2008-2009. Mas todos os números seguintes na citação dos autores estão errados. Nos arquivos de dados da POF, quando uma pessoa tem mais do que uma ocupação, há registros separados das características de cada ocupação. Verifica-se que há, na amostra, 101.053 registros de ocupação, mas o correspondente número de pessoas ocupadas é 84.730 . Há duas pessoas que informaram ter, cada uma, 8 ocupações.

Há 62.275 registros de trabalho como empregado, mas, devido à existência de pessoas com mais de um emprego, isso corresponde a 55.710 pessoas com algum trabalho como empregado e 53.670 pessoas que são empregados no seu trabalho principal. Entre as 55.710 pessoas com algum trabalho como empregado, 5.096

\footnotetext{
7 Consideramos como Auxílio Alimentação a parte da remuneração do empregado encontrada no Registro 15 da POF 2008-2009, com código 54015 (Quadro 54, incluindo os itens 01501 a 01504, denominados "auxilio/tíquete/cartão alimentação", "tíquetealimentação", "tíquete-refeição" e "cartão-refeição"). Cabe ressaltar que o eventual
}

informaram receber auxílio alimentação e entre as 53.670 pessoas que são empregados no seu trabalho principal, 5.040 recebem auxílio alimentação ${ }^{7}$. Na amostra, 9,4\% dos empregados no trabalho principal informaram receber o auxilio alimentação. Mas essa não é a porcentagem relevante. Deve-se calcular a porcentagem na população, usando os fatores de expansão de cada observação da amostra fornecidos pelo IBGE. Verifica-se, então, que, na população, 14,7\% dos que são empregados no trabalho principal recebem o auxílio alimentação.

Diante da lacuna existente na literatura sobre os efeitos do auxílio alimentação, este estudo objetiva analisar esses efeitos na distribuição da renda e nos índices antropométricos dos empregados beneficiados. Para isso serão utilizados os microdados da POF 2008-2009 disponibilizados pelo IBGE, juntamente com a respectiva documentação ${ }^{[7]}$.

Parece razoável admitir que o Auxílio Alimentação registrado na POF 2008-2009 seja associado ao PAT, pois toda empresa que concede esse benefício terá interesse em registrá-lo no PAT para obter a correspondente vantagem fiscal. No caso de funcionários públicos esse registro é importante para que haja garantia de que, do ponto de vista jurídico, o benefício não seja considerado parte do salário.

\section{O Auxílio Alimentação como parcela da renda familiar}

Vamos iniciar nossa análise considerando a renda familiar em toda a amostra da POF 20082009, que inclui 56.091 famílias ou Unidades de Consumo, representando uma população de 57,8 milhões de famílias, com 190,5 milhões de pessoas (3,295 pessoas por família). 
A Tabela 2 mostra os valores médios, por família e por pessoa, da renda total e de algumas de suas parcelas. Esses resultados foram obtidos a partir dos microdados da amostra, sempre levando em consideração os fatores de expansão da amostra. Trata-se, portanto, de médias ponderadas. O próprio IBGE já colocou todos os valores monetários em reais do dia de referência da
POF 2008-2009, que é 15 de janeiro de 2009. Para todos os tipos de rendimento, o valor per capita é obtido dividindo o valor do respectivo rendimento familiar pelo número de pessoas da família.

Tabela 2. A renda e suas parcelas

\begin{tabular}{|c|c|c|c|c|}
\hline Renda & $\begin{array}{l}\text { Renda média por } \\
\text { família(1) }^{(1)}\end{array}$ & $\begin{array}{l}\text { Renda média per } \\
\text { capita }^{(1)}\end{array}$ & $\begin{array}{l}\text { Participação na } \\
\text { renda total }(\%)\end{array}$ & $\begin{array}{l}\text { Índice de Gini } \\
\text { ou razão de } \\
\text { concentração }\end{array}$ \\
\hline Total & $2.763,47$ & 838,62 & 100,00 & 0,561 \\
\hline Renda do trabalho & $1.688,00$ & 512,26 & 61,08 & 0,566 \\
\hline Alugueis & 46,53 & 14,12 & 1,68 & 0,819 \\
\hline Aposentadorias e pensões do Instituto & & & & \\
\hline Nacional do Seguro Social & 281,83 & 85,53 & 10,20 & 0,480 \\
\hline Aposentadorias e pensões de & & & & \\
\hline funcionários públicos & 130,25 & 39,53 & 4,71 & 0,821 \\
\hline Transferências Federais & 19,51 & 5,92 & 0,71 & $-0,364$ \\
\hline Bolsa Família & 11,07 & 3,36 & 0,40 & $-0,562$ \\
\hline Benefício de Prestação Continuada & 8,29 & 2,52 & 0,30 & $-0,104$ \\
\hline Auxílio alimentação & 22,89 & 6,95 & 0,83 & 0,581 \\
\hline
\end{tabular}

Fonte: Elaborada pelos autores com base nos dados da POF 2008-2009, com a amostra de 56.091 famílias, representando uma população de 57,8 milhões de famílias.

(1) Em reais de 15/janeiro/2009.

Cabe ressaltar que as médias apresentadas na Tabela 2 se referem, sempre, a toda a população, considerando nulo o valor de uma parcela quando ela inexiste para uma família ou para uma pessoa. Se considerarmos apenas as famílias com valor positivo do auxílio alimentação (13,0\% do total), os valores médios por família para a renda total, para a renda do trabalho e para o auxílio alimentação são, respectivamente, $\mathrm{R} \$$ 4.480,18, R\$3.345,08 e R\$176,40.

A renda total inclui a renda do trabalho, a variação patrimonial, os alugueis, as transferências (incluindo as aposentadorias e pensões e os programas sociais federais) e o rendimento não monetário $^{8}$. Os dois principais componentes das

8 A composição completa pode ser vista na p. 188 da publicação do IBGE (2010)[8].
Transferências Federais são os rendimentos pagos pelo Programa Bolsa Família e o Benefício da Prestação Continuada (BPC). Os rendimentos do trabalho incluem os rendimentos da atividade como empregado, conta própria e empregador. $\mathrm{O}$ auxílio alimentação é parte do rendimento do trabalho de empregados.

Na última coluna da Tabela 2 encontramse o índice de Gini $(0,561)$ da renda familiar per capita (RFPC) e as razões de concentração de cada parcela? ${ }^{9}$ Parcelas com razão de concentração maior do que o índice de Gini são regressivas, contribuindo para aumentar a desigualdade da distribuição da RFPC. Por outro lado, quando a razão de concentração é menor do que o índice de

9 Uma apresentação do conceito de razão de concentração pode ser encontrada em Hoffmann (2014) ${ }^{[?]}$. 
Gini, a parcela é denominada progressiva, contribuindo para reduzir a desigualdade. Note-se que o rendimento de alugueis, geralmente associado a pessoas com maior patrimônio, é claramente regressivo, e o mesmo acontece com as aposentadorias e pensões de funcionários públicos. O Bolsa Família é um rendimento bastante bem focalizado nos pobres e, portanto, fortemente progressivo. Verifica-se que a razão de concentração do auxílio alimentação é um pouco maior do que o índice de Gini, mostrando que se trata de uma parcela regressiva da RFPC.

A maneira como as parcelas analisadas contribuem para a desigualdade da distribuição da RFPC pode ser visualizada na Figura 1, que mostra a curva de Lorenz da RFPC e as curvas de concentração de várias de suas parcelas. No eixo das abscissas mede-se a proporção acumulada das pessoas ordenadas conforme valores crescentes de sua RFPC. A curva de Lorenz é obtida considerando, na ordenada, a proporção acumulada da própria RFPC. A curva de concentração de uma parcela da RFPC é obtida medindo, na ordenada, a proporção acumulada do montante total da parcela na população. Uma curva de concentração que fica sempre abaixo da curva de Lorenz, como ocorre com os alugueis e as aposentadorias e pensões de funcionários públicos estatutários, mostra uma parcela concentrada em favor dos relativamente ricos, isto é, um componente regressivo da RFPC, cuja razão de concentração é necessariamente maior do que o índice de Gini. Se a curva de concentração fica acima do bissetor do primeiro quadrante, como ocorre com os rendimentos do Bolsa Familia, a razão de concentração é negativa, indicando uma parcela concentrada nos pobres.

Observa-se, na Figura 1, que a curva de concentração do auxílio alimentação cruza a curva de Lorenz em ponto que corresponde, aproximadamente, ao $3^{\circ}$ quartil da distribuição da RFPC. Nos primeiros $3 / 4$ da distribuição a curva de concentração fica abaixo da curva de Lorenz e para o quarto relativamente rico a curva de concentração fica acima da curva de Lorenz. Notase que os $20 \%$ mais pobres praticamente não se beneficiam do auxílio alimentação.

Figura 1. Curva de Lorenz da distribuição da renda familiar per capita e curvas de concentração de várias de suas parcelas

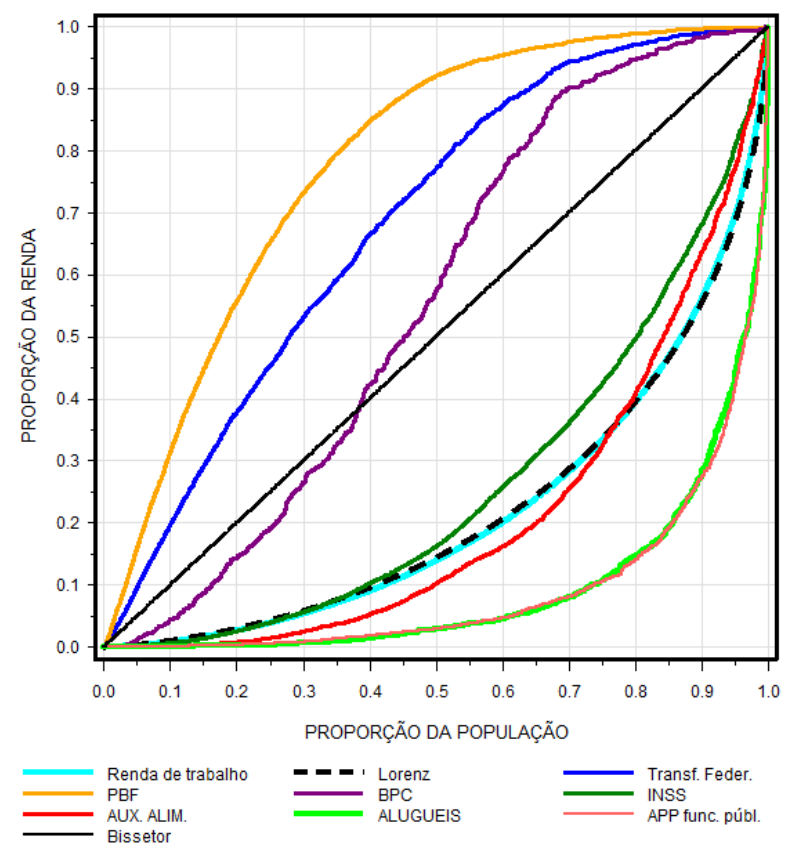

Fonte: Elaborada pelos autores com base nos microdados da POF 2008-2009, utilizando a amostra de 56.091 famílias, representando uma população de 190,5 milhões de pessoas.

A Figura 2 mostra a participação do auxilio alimentação na renda total de cada um dos décimos da distribuição da RFPC. No décimo mais pobre essa participação fica abaixo de um milésimo, superando um centésimo somente nos oitavo e nono décimos mais ricos. É interessante contrastar a Figura 2 com a Figura 3, que mostra a participação do Bolsa Família na renda total de cada décimo e evidencia o caráter fortemente progressivo desse rendimento. 
Figura 2. Participação do Auxílio Alimentação na renda em cada décimo da distribuição da renda familiar per capita

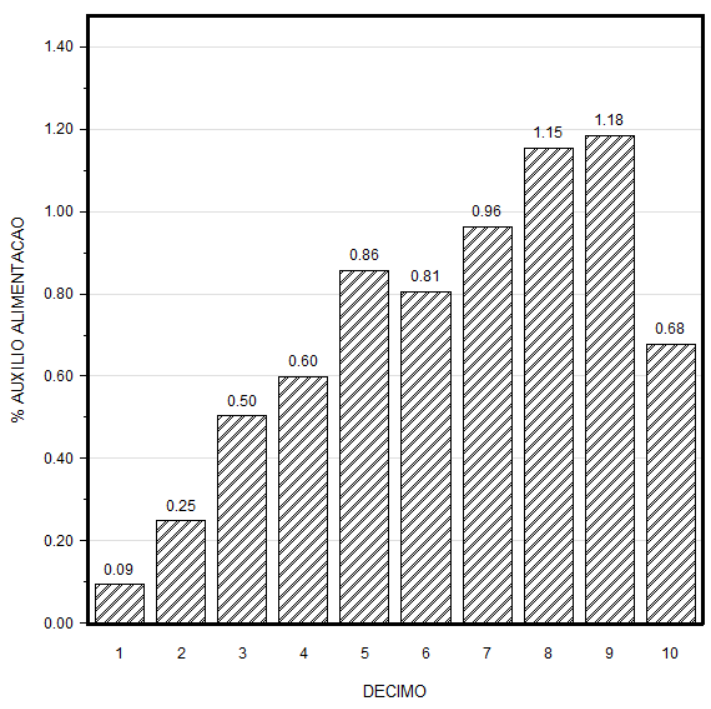

Fonte: Elaborada pelos autores com base nos dados da POF 2008-2009.

Figura 3. Participação das transferências do Programa Bolsa Família na renda em cada décimo da distribuição da renda familiar per capita

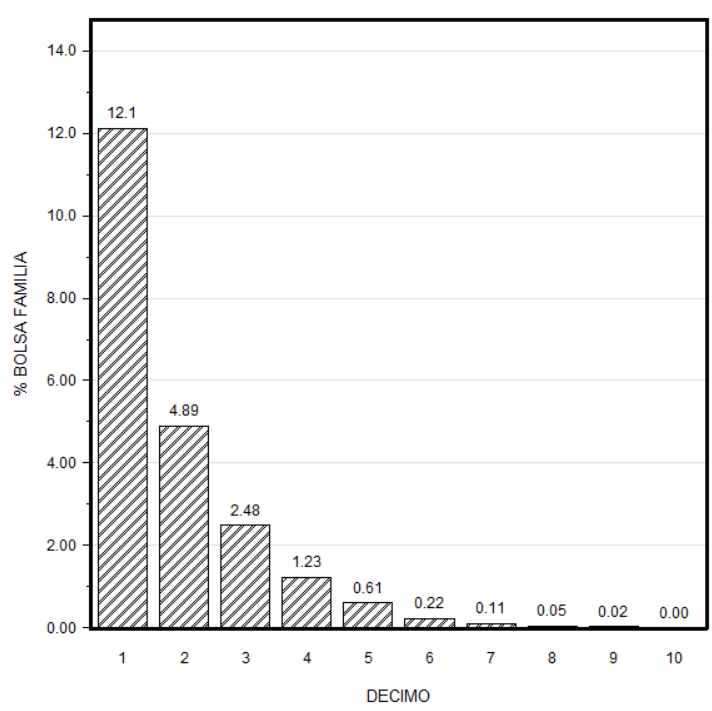

Fonte: Elaborada pelos autores com base nos dados da POF 2008-2009.
Enquanto o PAT é um programa criado para melhorar a alimentação dos trabalhadores, tendo como prioridade $\mathrm{O}$ atendimento aos trabalhadores que ganham até cinco salários mínimos, o Bolsa Família é destinado a famílias muito pobres, com o objetivo de combater a fome e a miséria ${ }^{10}$.O que se observa, por meio das Figuras 2 e 3, é que o Bolsa Família está focalizado nos muito pobres e, consequentemente, contribui para reduzir a desigualdade da distribuição de renda no Brasil. Algo muito diferente acontece com o PAT, cuja contribuição para a renda per capita é notoriamente mais baixa para os $20 \%$ mais pobres. É equivocado afirmar que o PAT é, também, um programa que contribui para reduzir a desigualdade da distribuição da renda.

\section{O Auxílio Alimentação na renda pessoal}

O auxílio alimentação é, tipicamente, parte do rendimento associado ao trabalho de uma pessoa como empregado ${ }^{11}$. Como uma pessoa pode receber o auxilio alimentação em mais de um emprego, para obter o valor desse rendimento por pessoa nos microdados da POF 2008-2009 é necessário somar, para cada pessoa, os valores associados aos vários empregos.

Entre as 190.159 pessoas da amostra da POF 2008-2009 há 5.255 que recebem auxílio alimentação. Isso corresponde, na população, a 8,9 milhões de pessoas que recebem o auxílio em um total de 190,5 milhões de pessoas. A proporção que recebe o auxílio alimentação é 4,66\%, embora na amostra seja apenas $2,76 \%$, porque o fator de expansão tende a ser maior para pessoas que estão no mercado formal de trabalho.

Há, na amostra, 84.730 pessoas ocupadas e, dessas, 55.710 têm alguma ocupação como empregado, sendo que 53.670 são empregados na sua atividade principal. Desses últimos, 5.040 recebem auxílio alimentação. $\mathrm{Na}$ população são

permite aos empregadores conceder o benefício a empregados com contrato suspenso ou interrompido. E permitida a continuidade de atendimento a trabalhadores dispensados, no período de transição para um novo emprego, limitado a um período de seis meses[ ${ }^{[6}$.
Atualmente (2017), com salario minimo nacional igual a $\mathrm{R} \$ 937,00$, podem receber a

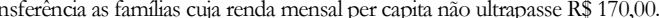

${ }^{11}$ Entre 5.255 pessoas da amostra da POF 2008-2009 que informaram receber auxílio alimentação há 74 que não tinham nenhuma ocupação. Isso é previsto na legislação, que 
8,67 milhões de empregados recebendo auxílio alimentação, correspondendo a $14,70 \%$ do total de 58,98 milhões de empregados.

Conforme os dados apresentados na Tabela 1, com informações do MTE, em 20082009 havia cerca de 14 milhões de trabalhadores beneficiados pelo PAT. Mas na POF 2008-2009 o número que recebe auxílio alimentação não chega a 9 milhões. Há, certamente, substancial subestimação do número de beneficiados na POF, já que deve ser frequente a omissão dessa informação nos questionários da pesquisa.

A Tabela 3 mostra a distribuição da população de trabalhadores que são empregados na sua atividade principal entre seis regiões do País. O estado de São Paulo foi destacado da região Sudeste, dada sua importância demográfica e econômica. Note-se que esse estado é a região de residência de 26,6\% dos empregados do País, de acordo com os dados da POF 2008-2009. Para cada região foi calculada a proporção de empregados que recebem auxílio alimentação. Observa-se que essa proporção é mais alta em SP $(22,3 \%)$ e nos demais estados do Sudeste $(19,3 \%)$ e fica abaixo de $8 \%$ nas regiões mais pobres (Norte e Nordeste).

O valor médio do benefício por empregado (considerando nulo o valor do benefício para aqueles que não o recebem) é fortemente condicionado pela proporção que recebe o auxílio alimentação. Assim, esse valor médio é maior (próximo de $\mathrm{R} \$ 30,00$ ) no Sudeste e é mais baixo no Norte e no Nordeste. O valor médio por empregado beneficiado varia menos: de R\$ 132,29 no Sul a R \$ 184,05 no Centro-Oeste. Note-se que a proporção de empregados que recebe o auxílio alimentação pode ser obtida dividindo o valor médio do benefício por empregado pelo seu valor médio por empregado beneficiado.

Tabela 3. Distribuição dos trabalhadores que são empregados no seu trabalho principal em 6 regiões, porcentagem que recebe o auxílio alimentação e valor médio do benefício, conforme dados da POF 2008-2009

\begin{tabular}{|c|c|c|c|c|c|c|}
\hline \multirow[b]{2}{*}{ Região } & \multicolumn{2}{|c|}{ Empregados } & \multirow[b]{2}{*}{ Salário médio(1) } & \multirow{2}{*}{$\begin{array}{l}\% \text { que recebe o } \\
\text { auxílio }\end{array}$} & \multicolumn{2}{|c|}{ Valor médio $(\mathrm{R} \$)$} \\
\hline & Número (mil) & $\%$ & & & Por empregado & $\begin{array}{c}\text { Por } \\
\text { beneficiado }\end{array}$ \\
\hline Norte & 3.607 & 6,1 & 950 & 7,3 & 12,92 & 177,56 \\
\hline Nordeste & 13.602 & 23,1 & 770 & 6,2 & 9,40 & 150,91 \\
\hline $\mathrm{MG}+\mathrm{ES}+\mathrm{RJ}$ & 12.451 & 21,1 & 1.248 & 19,3 & 29,92 & 154,84 \\
\hline $\mathrm{SP}$ & 15.704 & 26,6 & 1.384 & 22,3 & 32,50 & 145,69 \\
\hline Sul & 9.204 & 15,6 & 1.150 & 12,8 & 16,92 & 132,29 \\
\hline Centro-Oeste & 4.413 & 7,5 & 1.333 & 10,8 & 19,92 & 184,05 \\
\hline Total & 58.981 & 100,00 & 1.147 & 14,7 & 22,06 & 150,00 \\
\hline
\end{tabular}

Fonte: Elaborada pelos autores com base nos dados da POF 2008-2009.

(1) No trabalho principal. 
A Tabela 4 mostra os resultados de uma análise semelhante, considerando, agora, a divisão da população de empregados em 12 setores de atividade $^{12}$. O setor agrícola e os serviços domésticos se destacam pela menor proporção de beneficiados e pelo menor valor médio por empregado beneficiado (abaixo de R \$100,00).

Tabela 4. Distribuição dos trabalhadores que são empregados no seu trabalho principal em 12 setores de atividade, porcentagem que recebe o auxílio alimentação e valor médio do benefício, conforme dados da POF 2008-2009

\begin{tabular}{|c|c|c|c|c|c|c|}
\hline \multirow[b]{2}{*}{ Setor de Atividade } & \multicolumn{2}{|c|}{ Empregados } & \multirow[b]{2}{*}{$\begin{array}{l}\text { Salário } \\
\text { médio }^{(1)}\end{array}$} & \multirow[b]{2}{*}{$\begin{array}{c}\% \text { que recebe } \\
\text { o auxílio }\end{array}$} & \multicolumn{2}{|c|}{ Valor médio $(\mathrm{R} \$)$} \\
\hline & $\begin{array}{l}\text { Número } \\
\text { (mil) }\end{array}$ & $\%$ & & & $\begin{array}{c}\text { Por } \\
\text { empregado }\end{array}$ & $\begin{array}{c}\text { Por } \\
\text { beneficiado }\end{array}$ \\
\hline Agrícola & 4.677 & 7,9 & 474 & 2,4 & 2,42 & 99,68 \\
\hline Indústria de transformação & 9.430 & 16,0 & 1.221 & 17,1 & 19,88 & 115,98 \\
\hline Outras atividades industriais & 769 & 1,3 & 1.916 & 26,2 & 61,42 & 234,58 \\
\hline Construção & 3.075 & 5,2 & 875 & 12,6 & 16,31 & 129,88 \\
\hline Comércio e reparação & 9.483 & 16,1 & 867 & 13,3 & 15,90 & 119,19 \\
\hline Alojamento e alimentação & 2.322 & 3,9 & 648 & 13,5 & 15,84 & 117,21 \\
\hline $\begin{array}{l}\text { Transporte, armazenamento e } \\
\text { comunicação }\end{array}$ & 2.535 & 4,3 & 1.255 & 24,3 & 42,77 & 176,13 \\
\hline Administração pública & 4.668 & 7,9 & 2.438 & 18,0 & 34,78 & 193,16 \\
\hline $\begin{array}{l}\text { Educação, saúde e serviços } \\
\text { sociais }\end{array}$ & 7.165 & 12,2 & 1.571 & 14,8 & 19,45 & 131,48 \\
\hline Serviços domésticos & 7.045 & 11,9 & 352 & 5,5 & 5,43 & 99,63 \\
\hline Outros serviços & 1.807 & 3,1 & 1.031 & 11,3 & 16,87 & 149,35 \\
\hline Outras atividades & 6.005 & 10,2 & 1.642 & 27,9 & 56,35 & 202,25 \\
\hline Total & 58.981 & 100,00 & 1.147 & 14,7 & 22,06 & 150,00 \\
\hline
\end{tabular}

Fonte: Elaborada pelos autores com base nos dados da POF 2008-2009.

(1) No trabalho principal.

A Tabela 5 mostra os resultados obtidos considerando a divisão da população de empregados em nove grupamentos ocupacionais $^{13}$. Os militares (incluindo policiais e bombeiros) se destacam pela maior porcentagem de beneficiados, mas o maior valor médio do benefício para aqueles que o recebem ocorre no grupamento de Dirigentes. 
Tabela 5. Distribuição dos trabalhadores que são empregados no seu trabalho principal em 9 grupamentos ocupacionais, porcentagem que recebe o auxílio alimentação e valor médio do benefício, conforme dados da POF 20082009

\begin{tabular}{|c|c|c|c|c|c|c|}
\hline \multirow[b]{2}{*}{ Grupamento ocupacional } & \multicolumn{2}{|c|}{ Empregados } & \multirow{2}{*}{$\begin{array}{l}\text { Salário } \\
\text { médio }^{(1)}\end{array}$} & \multirow{2}{*}{$\begin{array}{l}\% \text { que } \\
\text { recebe o } \\
\text { auxílio }\end{array}$} & \multicolumn{2}{|c|}{ Valor médio $(\mathrm{R} \$)$} \\
\hline & $\begin{array}{l}\text { Número } \\
\text { (mil) }\end{array}$ & $\%$ & & & $\begin{array}{c}\text { Por } \\
\text { empregado }\end{array}$ & $\begin{array}{c}\text { Por } \\
\text { beneficiado }\end{array}$ \\
\hline Dirigentes & 2.264 & 3,8 & 2.941 & 22,1 & 51,03 & 230,63 \\
\hline Profissionais & 5.013 & 8,5 & 3.167 & 17,4 & 30,91 & 178,05 \\
\hline Militares/Policiais/Bombeiros & 578 & 1,0 & 2.789 & 26,1 & 43,68 & 167,60 \\
\hline Técnicos & 4.688 & 7,9 & 1.745 & 22,9 & 44,64 & 195,04 \\
\hline Serviços administrativos & 7.638 & 13,0 & 1.121 & 21,9 & 36,38 & 166,01 \\
\hline Trabalhadores dos serviços & 16.202 & 27,5 & 580 & 11,8 & 13,79 & 117,13 \\
\hline Vendedores/serv.do comércio & 4.400 & 7,5 & 703 & 10,0 & 11,79 & 118,14 \\
\hline Trab. Indústria/manutenção & 13.822 & 23,4 & 901 & 14,2 & 17,02 & 119,89 \\
\hline Trabalhadores agrícolas & 4.375 & 7,4 & 412 & 2,2 & 1,69 & 78,03 \\
\hline Total & 58.981 & 100,00 & 1.147 & 14,7 & 22,06 & 150,00 \\
\hline
\end{tabular}

Fonte: Elaborada pelos autores com base nos dados da POF 2008-2009.

(1) No trabalho principal.

Verifica-se que nas Tabelas 4 e 5 há correlação muito forte entre o salário médio dos empregados e o valor médio do auxílio por beneficiado $(r=0,820$ na Tabela 4 e $r=0,816$ na Tabela 5), ambas significativas ao nível de 1\%. $\mathrm{O}$ auxílio médio é maior nos setores e nas ocupações onde o empregado já ganha mais. Isso não ocorre na Tabela 3, na qual essa correlação é quase nula $(r=0,014)$. Mas nessas três tabelas há forte correlação positiva entre o salário médio e a porcentagem de empregados que recebe o auxílio alimentação ( $r$ igual a $0,797,0,723$ e 0,712 nas Tabelas 3, 4 e 5, respectivamente) e a correlação entre o salário médio e o valor médio do auxílio por empregado é positiva e estatisticamente significativa ao nível de 5\% ( $r$ igual a 0,853, 0,747 e 0,789 para as Tabelas 3, 4 e 5, respectivamente). Isso faz com que o salário médio dos empregados que recebem o auxilio $(\mathrm{R} \$ 2.027)$ supere o dobro do salário médio daqueles que não recebem o auxílio ( $\mathrm{R}$ \$ 995), confirmando o caráter regressivo desse benefício, já constatado na seção anterior.
Nas normas do PAT consta que deve ser dada "prioridade ao atendimento dos trabalhadores de baixa renda"14, assim considerados aqueles com salário mensal equivalente a até cinco salários mínimos ${ }^{15}$. Tal limite é relativamente elevado e não faz com que o auxílio alimentação seja focalizado nos trabalhadores pobres. Dados da Pesquisa Nacional por Amostra de Domicílios (PNAD) ${ }^{[8]}$ de 2015 indicam que para 92,6\% dos empregados, no Brasil, com informação do rendimento no trabalho principal, esse rendimento não ultrapassava cinco salários mínimos. Limitando a análise aos empregados formais (com carteira de trabalho assinada) essa proporção é 90,7\%. É certo que essa porcentagem deve estar superestimada devido à subdeclaração dos rendimentos na PNAD. Mas é importante ter em mente que o fato de mais de $80 \%$ dos empregados beneficiados pelo PAT receberem salários iguais ou menores do que 5 salários mínimos não significa que o programa esteja focalizado nos relativamente pobres. 
No caso dos funcionários públicos, a concessão de cartões alimentação e refeição provavelmente se deu mais como meio de aumentar a remuneração dos funcionários ativos, sem onerar os cofres públicos com aumentos no valor de aposentadorias e pensões, e não, essencialmente, para "melhoria da situação nutricional dos trabalhadores, visando a promover sua saúde e prevenir as doenças profissionais" ${ }^{\text {16. }}$.

Os dados da POF 2008-2009 mostram que o Auxílio Alimentação não pode ser defendido como um benefício focalizado nos trabalhadores pobres. Ao contrário: vimos, na seção 2, que se trata de uma parcela do rendimento total das famílias cuja razão de concentração é um pouco maior do que o índice de Gini da distribuição da RFPC, caracterizando um componente regressivo da renda. Os resultados apresentados nesta seção confirmam o caráter regressivo desse benefício. Mas o Auxílio Alimentação pode, sim, ser defendido por facilitar ao trabalhador obter boa alimentação no local de trabalho ou próximo dele, evitando deslocamentos excessivos e promovendo a produtividade do trabalho.

\section{O Auxílio Alimentação e o IMC}

O PAT já tem mais de quatro décadas. Nesse período a renda real média do trabalhador brasileiro cresceu substancialmente. Houve redução da desigualdade da distribuição da renda, o que significa que a renda média dos relativamente pobres cresceu mais do que a média geral. As despesas com alimentação pesam cada vez menos nos orçamentos familiares. Comida menos escassa e o enorme crescimento de atividades sedentárias fez com que o sobrepeso e a obesidade se tornassem importantes problemas da saúde pública no Brasil.

Dados da POF 2008-2009 mostram que a RFPC é um condicionante fundamental da probabilidade de ocorrência de sobrepeso e obesidade, que essa relação não é linear e é muito diferente para homens e para mulheres ${ }^{[10]}$. Para homens a probabilidade de ser obeso cresce fortemente com a RFPC até o estrato de mais de $\mathrm{R} \$ 2.100$ a $\mathrm{R} \$ 3.500$, enquanto para mulheres a associação positiva entre RFPC e probabilidade de obesidade é bem mais fraca e se torna negativa a partir do estrato de mais de $\mathrm{R} \$ 1.120$ a $\mathrm{R} \$ 1.400$ (ver Figuras 6 e 8 em Hoffmann, 2012 ${ }^{[10]}$.

Uma vez que o Auxílio Alimentação é parte da renda do trabalhador, é claro que ele deve afetar seu Índice de Massa Corporal (IMC) e, consequentemente, a probabilidade de apresentar sobrepeso ou obesidade. O problema relevante é saber se o fato de essa parte do rendimento estar diretamente associada ao consumo ou aquisição de alimentos tem um efeito específico sobre o IMC. Para captar esse efeito é fundamental controlar os demais fatores que afetam o IMC, com especial atenção para a renda.

A revisão da literatura permite destacar artigo de Veloso, Santana e Oliveira (2007) $)^{[11]}$ como o único concluindo que "Programas de alimentação para trabalhadores contribuíram para o ganho de peso e sobrepeso". Foi utilizada uma amostra de 10.368 trabalhadores da Bahia, com dados de pelo menos duas consultas médicas entre 14/11/1995 e 31/03/2000. A relação entre o ganho de peso observado e haver ou não programa de alimentação foi avaliada controlando sexo, idade, intensidade física da ocupação e nível socioeconômico. Não houve controle específico da renda e foram considerados apenas três estratos (alto, médio e baixo) de nível socioeconômico (definido com base em grupos ocupacionais e na escolaridade do trabalhador). É claro que isso não possibilita o controle apropriado do efeito não linear da renda sobre o IMC. Assim, provavelmente a associação entre ganho de peso e sobrepeso e o trabalhador ser beneficiário de programa de alimentação se deve ao fato de a

16 Art. 1o da Portaria no 3, de 1o de março de 2002, MTE, Secretaria de Inspeção do Trabalho. 
renda ser maior para os trabalhadores que recebem o auxílio.

Para analisar o eventual efeito do auxílio alimentação sobre o IMC nos dados da POF 20082009 vamos considerar os dados antropométricos (inclusive valores imputados pelo IBGE) apenas para pessoas com 20 anos ou mais de idade, excluindo mulheres grávidas e pessoas com idade estimada. O escore $Z$ do $\mathrm{IMC}^{17}$ é usado como variável dependente. Foram excluídas, também, as pessoas com escore $Z$ da altura fora do intervalo de -6 a 6 e aquelas com escore $Z$ do IMC fora do intervalo de -5 a 5 , pois se trata de valores biologicamente implausíveis. Tendo em vista, ainda, os controles que serão utilizados, foi necessário excluir as pessoas sem informação de escolaridade ou de cor da pele. Também foram excluídos os que declararam cor "indígena", devido a seu pequeno número na amostra.

Com esses filtros a amostra ficou reduzida a 118.303 pessoas $^{18}$, dos quais 47.929 são empregados no trabalho principal (21.108 mulheres e 26.821 homens). Como o auxílio alimentação é destinado, essencialmente, aos empregados, na análise apresentada a seguir serão destacados os resultados obtidos utilizando a amostra de pessoas dessa categoria.

A amostra de 47.929 empregados representa uma população de 52.896 .482 pessoas dessa categoria (23.990 mil mulheres e 28.906 mil homens). A Tabela 6 mostra a distribuição dessa população conforme faixas de escolaridade, categorias de cor, estratos de RFPC, ter residência em área urbana ou área metropolitana, número de crianças de 0 a 4 anos na família, número de crianças de 5 a 9 anos na família, apresentar sobrepeso ou ser obeso, sempre destacando mulheres e homens ${ }^{19}$.

Foram consideradas com sobrepeso pessoas com IMC maior ou igual a $25 \mathrm{~kg} / \mathrm{m}^{2}$ e as com IMC maior ou igual a $30 \mathrm{~kg} / \mathrm{m}^{2}$ foram classificadas como obesas. Observa-se que a proporção de empregados nos dois estratos de escolaridade mais elevada é substancialmente maior para mulheres do que para homens. A proporção com sobrepeso é maior entre homens, mas a proporção com obesidade é maior entre mulheres.

17 A descrição do cálculo do escore $Z$ do IMC pode ser encontrada em Hoffmann $(2012)^{[10]}$.

18 Amostra um pouco menor do que a utilizada em Hoffmann (2012)[10], pois lá foram incluídas as pessoas com idade estimada.

19 A Tabela 6 é semelhante à Tabela 1 em Hoffmann (2012) ${ }^{[10]}$, mas limitada aos empregados. Cabe ressaltar que naquele artigo foi incluída na categoria "metropolitano" a população de todas as capitais de estados, enquanto no presente artigo se respeita a definição do IBGE. 
Tabela 6. População de empregados correspondente à amostra analisada e sua distribuição em várias classificações, conforme o sexo

\begin{tabular}{|c|c|c|c|c|c|c|}
\hline \multirow{2}{*}{ Classificação/Categoria } & \multicolumn{2}{|c|}{ Total } & \multicolumn{2}{|c|}{ Mulheres } & \multicolumn{2}{|c|}{ Homens } \\
\hline & $\mathrm{n}-\mathrm{o}(1000)$ & $\%$ & no $(1000)$ & $\%$ & no $(1000)$ & $\%$ \\
\hline Total & 52.896 & 100,00 & 23.990 & 100,00 & 28.906 & 100,00 \\
\hline \multicolumn{7}{|l|}{ Escolaridade: } \\
\hline 0 & 1.917 & 3,62 & 628 & 2,62 & 1.289 & 4,46 \\
\hline $1-2$ & 2.541 & 4,80 & 863 & 3,60 & 1.678 & 5,80 \\
\hline $3-4$ & 6.655 & 12,58 & 2.768 & 11,54 & 3.887 & 13,45 \\
\hline $5-8$ & 11.697 & 22,11 & 4.912 & 20,48 & 6.784 & 23,47 \\
\hline $9-10$ & 3.524 & 6,66 & 1.447 & 6,03 & 2.077 & 7,19 \\
\hline 11 & 15.812 & 29,89 & 7.344 & 30,61 & 8.469 & 29,30 \\
\hline $12-14$ & 3.853 & 7,28 & 2.015 & 8,40 & 1.837 & 6,36 \\
\hline$\geq 15$ & 6.899 & 13,04 & 4.013 & 16,73 & 2.886 & 9,98 \\
\hline \multicolumn{7}{|l|}{ Cor } \\
\hline Branco & 26.491 & 50,08 & 12.445 & 51,87 & 14.046 & 48,59 \\
\hline Preto & 4.851 & 9,17 & 2.162 & 9,01 & 2.689 & 9,30 \\
\hline Pardo & 21.273 & 40,22 & 9.239 & 38,51 & 12.034 & 41,63 \\
\hline Amarelo & 281 & 0,53 & 144 & 0,60 & 137 & 0,47 \\
\hline \multicolumn{3}{|l|}{ REPC } & 991 & 4.13 & 1.589 & 550 \\
\hline$(140 ; 280]$ & 7.288 & 13,78 & 3.088 & 12,87 & 4.199 & 14,53 \\
\hline$(280 ; 560]$ & 14.263 & 26,96 & 6.400 & 26,68 & 7.863 & 27,20 \\
\hline$(560 ; 840]$ & 9.521 & 18,00 & 4.351 & 18,14 & 5.170 & 17,88 \\
\hline$(840 ; 1.120]$ & 5.730 & 10,83 & 2.703 & 11,27 & 3.028 & 10,47 \\
\hline$(1.120 ; 1.400]$ & 3.729 & 7,05 & 1.796 & 7,48 & 1.933 & 6,69 \\
\hline$(1.400 ; 2.100]$ & 4.403 & 8,32 & 2.071 & 8,63 & 2.332 & 8,07 \\
\hline$(2.100 ; 3.500]$ & 3.051 & 5,77 & 1.429 & 5,96 & 1.621 & 5,61 \\
\hline (3.500; 5.600] & 1.439 & 2,72 & 704 & 2,94 & 734 & 2,54 \\
\hline$>5.600$ & 894 & 1,69 & 457 & 1,91 & 437 & 1,51 \\
\hline Urbano & 47.330 & 89,48 & 22.088 & 92,07 & 25.242 & 87,32 \\
\hline Metropolitano & 17.364 & 32,83 & 8.222 & 34,27 & 9.142 & 31,63 \\
\hline \multicolumn{7}{|l|}{ Criança de 0 a 4 anos: } \\
\hline 0 & 40.930 & 77,38 & 18.888 & 78,73 & 22.042 & 76,25 \\
\hline 1 & 10.071 & 19,04 & 4.367 & 18,20 & 5.704 & 19,73 \\
\hline 2 ou mais & 1.895 & 3,58 & 735 & 3,06 & 1.160 & 4,01 \\
\hline \multicolumn{7}{|l|}{ Criança de 5 a 9 anos: } \\
\hline 0 & 39.686 & 75,03 & 17.979 & 74,94 & 21.707 & 75,09 \\
\hline 1 & 10.682 & 20,19 & 4.859 & 20,26 & 5.822 & 20,14 \\
\hline 2 ou mais & 2.529 & 4,78 & 1.152 & 4,80 & 1.377 & 4,76 \\
\hline Com sobrepeso & 24.800 & 46,88 & 10.223 & 42,61 & 14.577 & 50,43 \\
\hline Obeso & 6.720 & 12,70 & 3.253 & 13,56 & 3.467 & 12,00 \\
\hline Recebe auxílio alimentação & 8.246 & 15,59 & 3.411 & 14,22 & 4.835 & 16,73 \\
\hline
\end{tabular}

Fonte: Elaborada pelos autores com base nos dados da POF 2008-2009.

Todas as equações analisadas adiante são estimadas com ponderação pelo fator de expansão fornecido pelo IBGE e levando em consideração a estrutura complexa da amostra da POF 20082009.
A análise será feita separadamente para homens e para mulheres. Ao fazer uma análise de regressão com o escore $Z$ do IMC como variável dependente, se agregarmos os dois sexos, é claramente insuficiente usar uma variável binária 
para controlar o efeito de sexo, pois a diferença não ocorre apenas no nível da função. Como o efeito de cada estrato de renda é diferente conforme o sexo, seria necessário controlar a interação de sexo com a renda. Se incluirmos as interações de sexo com todas as demais variáveis explanatórias, a regressão com a amostra agregada será equivalente a fazer regressões separadas para homens e para mulheres, mas nesse caso a equação estimada com todas aquelas interações fica complicada, tornando-se conveniente a estimação de equações distintas para cada sexo.
A Tabela 7 mostra os resultados obtidos ajustando equações do escore $Z$ do IMC contra uma binária que indica se o empregado é ou não beneficiário do auxilio alimentação, a idade da pessoa e seu quadrado. $\mathrm{Na}$ ausência de qualquer outro controle além da idade, as equações estimadas indicam que o auxílio alimentação tem forte efeito positivo sobre o IMC de homens, mas não afeta significativamente o IMC de mulheres empregadas.

Tabela 7. Equações de regressão do Score $Z$ do IMC de empregados em função da idade e de uma variável binária para auxílio alimentação, para mulheres e para homens, com 20 ou mais anos de idade, no Brasil, 2008-2009

\begin{tabular}{|c|c|c|c|c|}
\hline \multirow{2}{*}{ Variável explanatória } & \multicolumn{2}{|c|}{ Mulheres } & \multicolumn{2}{|c|}{ Homens } \\
\hline & Coeficiente & $p^{(1)}$ & Coeficiente & $p^{(1)}$ \\
\hline Constante & $-1,1070$ & * & $-1,1226$ & * \\
\hline Idade (em décadas) & 0,7957 & * & 0,8914 & * \\
\hline Idade ao quadrado & $-0,0674$ & * & $-0,0890$ & * \\
\hline Binária para Auxílio Alimentação & 0,0365 & $38,0 \%$ & 0,1319 & * \\
\hline Teste $F$ & 261,27 & * & 254,24 & * \\
\hline Coef. de Determ. $\left(R^{2}\right)$ & \multicolumn{2}{|c|}{0,0765} & \multicolumn{2}{|c|}{0,0563} \\
\hline Número de observações & \multicolumn{2}{|c|}{21.108} & \multicolumn{2}{|c|}{26.821} \\
\hline
\end{tabular}

Fonte: Elaborada pelos autores com base nos dados da POF 2008-2009.

(1) Probabilidade caudal do teste da hipótese de nulidade do parâmetro ou, no caso do teste $F$, nulidade de todos os coeficientes de regressão. $\mathrm{O}$ asterisco indica que essa probabilidade é inferior a $0,1 \%$.

Para captar o efeito da RFPC foram incluídas, no modelo de regressão, variáveis binárias que assumem valor 1 quando a pessoa pertence a um dos estratos de RFPC enumerados na Tabela 6 e assumem valor zero em caso contrário. Adotando o estrato mais pobre como base, são incluídas 9 variáveis binárias e o respectivo coeficiente, na equação estimada, indicará o quanto, em média, o $Z$ do IMC das pessoas dos demais estratos difere do observado no estrato mais pobre.

Os resultados apresentados na Tabela 8 mostram que para os homens a renda mais alta está fortemente associada ao aumento do IMC, ao passo que para as mulheres os efeitos significativos indicam um IMC mais baixo nos dois estratos de maior renda, em comparação com o estrato mais pobre.

Um resultado importante é que, para os homens, a mera inclusão do controle da RFPC faz quase desaparecer a associação entre o fato de receber auxílio alimentação e o escore $Z$ do IMC.

Por outro lado, para as mulheres, com o controle da RFPC o efeito do auxílio alimentação se torna significativo ao nível de $10 \%$. Note-se que o controle do efeito da renda é feito usando a renda total informada, incluindo o valor do auxílio alimentação. Dessa maneira, o efeito captado por meio da binária para o auxílio alimentação deverá 
ser apenas o efeito, sobre o $Z$ do IMC, do fato de receber parte da renda diretamente na forma de alimentos ou especificamente destinada à compra de alimentos.

Tabela 8. Equações de regressão do Score $Z$ do IMC de empregados em função da idade, de variáveis binárias para 10 estratos de RFPC e de uma variável binária para auxílio alimentação, para mulheres e para homens, com 20 ou mais anos de idade, no Brasil, 2008-2009

\begin{tabular}{|c|c|c|c|c|}
\hline \multirow{2}{*}{ Variável explanatória } & \multicolumn{2}{|c|}{ Mulheres } & \multicolumn{2}{|c|}{ Homens } \\
\hline & Coeficiente & $p^{(1)}$ & Coeficiente & $p^{(1)}$ \\
\hline Constante & $-1,1085$ & $*$ & $-1,4998$ & $*$ \\
\hline Idade (em décadas) & 0,7924 & * & 0,9095 & * \\
\hline Idade ao quadrado & $-0,0660$ & * & $-0,0933$ & * \\
\hline \multicolumn{5}{|l|}{ RFPC: } \\
\hline Mais de 140 a 280 & 0,0187 & $73,2 \%$ & 0,1748 & $*$ \\
\hline Mais de 280 a 560 & 0,0237 & $64,3 \%$ & 0,3078 & * \\
\hline Mais de 560 a 840 & 0,0420 & $45,2 \%$ & 0,4387 & * \\
\hline Mais de 840 a 1.120 & $-0,0474$ & $43,4 \%$ & 0,5478 & * \\
\hline Mais de 1.120 a 1.400 & $-0,0656$ & $31,7 \%$ & 0,5947 & * \\
\hline Mais de 1.400 a 2.100 & $-0,0100$ & $87,9 \%$ & 0,6230 & * \\
\hline Mais de 2.100 a 3.500 & $-0,0370$ & $57,9 \%$ & 0,6092 & * \\
\hline Mais de 3.500 a 5.600 & $-0,2854$ & * & 0,6236 & * \\
\hline Mais de 5.600 & $-0,3125$ & $0,7 \%$ & 0,6210 & $*$ \\
\hline Binária para Auxílio Alimentação & 0,0715 & $7,7 \%$ & 0,0206 & $45,2 \%$ \\
\hline Teste $F$ & 69,48 & * & 108,86 & * \\
\hline Coef. de determ. $\left(R^{2}\right)$ & \multicolumn{2}{|c|}{0,0808} & \multicolumn{2}{|c|}{0,0831} \\
\hline Número de observações & \multicolumn{2}{|c|}{21.108} & \multicolumn{2}{|c|}{26.821} \\
\hline
\end{tabular}

Fonte: Elaborada pelos autores com base nos dados da POF 2008-2009.

(1) Probabilidade caudal do teste da hipótese de nulidade do parâmetro ou, no caso do teste $F$, nulidade de todos os coeficientes de regressão. $O$ asterisco indica que essa probabilidade é inferior a $0,1 \%$.

A Tabela 9 mostra os resultados obtidos controlando a região de residência e todas as classificações apresentadas na Tabela 6.

Em todos os casos são usadas variáveis binárias para distinguir as categorias, adotando como base a Região Nordeste, cor branca, os estratos de escolaridade e RFPC mais baixos, área rural e ausência, na família, de crianças de 0 a 4 anos ou de 5 a 9 anos.
O efeito do auxílio alimentação permanece claramente não significativo para homens e significativo ao nível de 10\% para mulheres. 
Tabela 9. Equações de regressão múltipla estimadas para avaliar os condicionantes do escore Z do IMC de mulheres e de homens empregados, com 20 ou mais anos de idade, no Brasil, 2008-2009, incluindo, como variável explanatória, uma variável binária para o recebimento de Auxílio Alimentação

\begin{tabular}{|c|c|c|c|c|}
\hline \multirow{2}{*}{ Variável explanatória } & \multicolumn{2}{|c|}{ Mulheres } & \multicolumn{2}{|c|}{ Homens } \\
\hline & Coeficiente & $p^{(1)}$ & Coeficiente & $p^{(1)}$ \\
\hline Constante & $-1,0654$ & * & $-1,7736$ & * \\
\hline Idade (em décadas) & 0,7721 & * & 0,8907 & * \\
\hline Idade ao quadrado' & $-0,0662$ & * & $-0,0885$ & * \\
\hline \multicolumn{5}{|l|}{ Escolaridade: } \\
\hline $1-2$ & 0,0033 & $96,6 \%$ & 0,0229 & $63,8 \%$ \\
\hline $3-4$ & $-0,0136$ & $83,0 \%$ & 0,1973 & * \\
\hline $5-8$ & $-0,0977$ & $10,8 \%$ & 0,2382 & * \\
\hline $9-10$ & $-0,1330$ & $5,9 \%$ & 0,2086 & * \\
\hline 11 & $-0,2125$ & $*$ & 0,2419 & * \\
\hline $12-14$ & $-0,3178$ & * & 0,1796 & $0,6 \%$ \\
\hline$\geq 15$ & $-0,3496$ & $*$ & 0,1458 & $0,9 \%$ \\
\hline \multicolumn{5}{|l|}{ Cor: } \\
\hline Preto & 0,1235 & $0,7 \%$ & $-0,0642$ & $6,9 \%$ \\
\hline Pardo & 0,0329 & $19,9 \%$ & $-0,0522$ & $1,6 \%$ \\
\hline Amarelo & $-0,1493$ & $29,1 \%$ & $-0,2329$ & $12,1 \%$ \\
\hline \multicolumn{5}{|l|}{ Região: } \\
\hline Norte & 0,0423 & $17,6 \%$ & 0,0519 & $6,7 \%$ \\
\hline $\mathrm{MG}+\mathrm{ES}+\mathrm{RJ}$ & 0,0223 & $44,9 \%$ & $-0,0525$ & $4,8 \%$ \\
\hline SP & 0,0066 & $87,2 \%$ & 0,0282 & $41,1 \%$ \\
\hline Sul & 0,0616 & $5,4 \%$ & 0,0829 & $0,4 \%$ \\
\hline Centro-Oeste & 0,0205 & $50,8 \%$ & 0,0538 & $4,9 \%$ \\
\hline $\begin{array}{l}\text { RFPC: } \\
\quad \text { Mais de } 140 \text { a } 280\end{array}$ & 0,0705 & \multicolumn{2}{|c|}{ RFPC: } & * \\
\hline Mais de 280 a 560 & 0,1389 & $1,2 \%$ & 0,2526 & * \\
\hline Mais de 560 a 840 & 0,2144 & $*$ & 0,3626 & * \\
\hline Mais de 840 a 1.120 & 0,1643 & $1,2 \%$ & 0,4703 & $*$ \\
\hline Mais de 1.120 a 1.400 & 0,1839 & $1,1 \%$ & 0,5163 & $*$ \\
\hline Mais de 1.400 a 2.100 & 0,2751 & $*$ & 0,5508 & * \\
\hline Mais de 2.100 a 3.500 & 0,2938 & $*$ & 0,5440 & * \\
\hline Mais de 3.500 a 5.600 & 0,0726 & $44,0 \%$ & 0,5686 & * \\
\hline Mais de 5.600 & 0,0850 & $46,8 \%$ & 0,5851 & * \\
\hline Urbano & $-0,0494$ & $12,4 \%$ & 0,1348 & * \\
\hline Metropolitano & $-0,0008$ & $98,0 \%$ & $-0,0092$ & $72,5 \%$ \\
\hline \multicolumn{5}{|l|}{ Criança de 0 a 4 anos: } \\
\hline 1 & 0,0771 & * & 0,0695 & $0,3 \%$ \\
\hline 2 ou mais & 0,2224 & * & 0,0886 & $6,2 \%$ \\
\hline \multicolumn{5}{|l|}{ Crianca de 5 a 9 anos: } \\
\hline 1 & 0,1021 & * & 0,0715 & $0,4 \%$ \\
\hline 2 ou mais & 0,0544 & $31,8 \%$ & 0,0546 & $20,6 \%$ \\
\hline Binária para Auxílio Alimentação & 0,0684 & $8,1 \%$ & 0,0091 & $74,7 \%$ \\
\hline Teste $F$ & 30,42 & $*$ & 48,63 & $*$ \\
\hline \multirow{2}{*}{$\begin{array}{l}\text { Coeficiente de determinação }\left(R^{2}\right) \\
\text { Número de observações }\end{array}$} & \multicolumn{2}{|c|}{0,0924} & \multicolumn{2}{|c|}{0,0945} \\
\hline & \multicolumn{2}{|c|}{21.108} & \multicolumn{2}{|c|}{26.821} \\
\hline
\end{tabular}

Fonte: Elaborada pelos autores com base nos dados da POF 2008-2009.

(1) Probabilidade caudal do teste da hipótese de nulidade do parâmetro ou, no caso do teste $F$, nulidade de todos os coeficientes de regressão. $O$ asterisco indica que essa probabilidade é inferior a $0,1 \%$. 
Note-se que a RFPC se revela um condicionante muito mais forte do IMC para os homens do que para as mulheres. Para os homens o escore $Z$ do IMC tende a crescer sistematicamente com a renda, ao passo que para as mulheres esse escore tende a ser mais alto para estratos intermediários, mas para os dois estratos de maior RFPC seu valor médio não é estatisticamente diferente daquele do estrato mais pobre. Já o efeito da presença de crianças de 0 a 9 anos é, geralmente, mais forte para as mulheres, provavelmente por razões biológicas e, também, devido ao papel atribuído às mulheres no cuidado com os filhos.

Para avaliar o perigo de detectar um falso efeito do auxílio alimentação devido ao controle inapropriado da renda, fizemos o seguinte exercício: ajustamos uma modelo como o da Tabela 9 para a amostra agregada de mulheres e homens, incluindo uma variável binaria para sexo do empregado; o efeito do auxílio alimentação não foi significativo (valor $p$ igual a 17,0\%); excluindo do modelo as binárias que distinguem os 10 estratos de RFPC, mas mantendo todos os demais controles, o efeito do auxílio alimentação se torna significativo ao nível de $1 \%$; esse efeito é falso, pois capta o efeito da renda mais alta (devido à forte associação entre renda e IMC dos homens e ao fato de os empregados que recebem o auxílio alimentação terem, em média, renda muito mais alta).

Foram estimados vários outros modelos para o $Z$ do IMC, adicionando, como controles, variáveis binárias para distinguir os 12 setores de atividade da Tabela 4, os 9 grupamentos ocupacionais da Tabela 5 e estratos do valor do salário do empregado. Também foram ajustados modelos de lógite para a probabilidade de o empregado ter sobrepeso ou ser obeso, utilizando os mesmos controles. No caso dos homens, o efeito do auxílio alimentação é sempre claramente não significativo, indicando que, quando a influência do nível de renda é apropriadamente controlada, o fato de receber auxílio alimentação não tem efeito sobre o IMC ou sobre a probabilidade de ter sobrepeso ou ser obeso.

Os resultados são mais complexos no caso das mulheres. Nos modelos para o escore $Z$ do IMC o efeito do auxílio alimentação é, em geral, significativo ao nível de 10\%. Já um modelo de lógite para a probabilidade de a mulher empregada ser obesa com as mesmas variáveis explanatórias da Tabela 9 mostra um efeito positivo para o auxílio alimentação, agora com probabilidade caudal do teste de nulidade igual a 1,9\%. Incluindo variáveis binárias para setores de atividade e para grupamentos de ocupação, a probabilidade caudal do teste de nulidade do efeito do auxílio alimentação se reduz a 1,5\%. Há, portanto, indicações de que, em algumas situações, o auxilio alimentação contribui para a obesidade das mulheres.

\section{CONCLUSÃO}

Os dados da POF 2008-2009 registram o auxílio alimentação como uma parcela da remuneração dos empregados, beneficiando $14,7 \%$ dos trabalhadores dessa categoria. Considerando a distribuição da população de empregados pelas regiões do País ou sua divisão em 12 setores ou 9 grupamentos ocupacionais, verifica-se, nos três casos, que há correlação positiva e significativa entre o salário médio e o valor médio desse auxilio por empregado. Analisando a participação do auxílio alimentação na RFPC, considerando toda a população, constata-se que ele contribui para reforçar a desigualdade da distribuição da renda no País. A Figura 2 mostra que a participação desse auxílio na RFPC tende a crescer do décimo mais pobre até o nono décimo da distribuição. $\mathrm{O}$ auxílio alimentação certamente pode facilitar ao trabalhador o acesso a refeições no local de trabalho ou próximo dele, mas os dados da POF 2008-2009 não mostram que o benefício esteja focalizado nos relativamente pobres. 
No que se refere ao efeito do auxílio alimentação sobre o IMC ou sobre a incidência de sobrepeso ou obesidade, verifica-se que, quando devidamente controlado o efeito da renda, não se detecta qualquer efeito do auxílio sobre aquelas características antropométricas dos homens empregados. Já no caso das mulheres empregadas, os dados indicam que há situações nas quais o auxílio alimentação favorece a obesidade.

\section{REFERÊNCIAS}

[1] Departamento Intersindical de Estatísticas e Estudos Socioeconômicos - DIEESE. Projeto de Proposta de reformulação do Programa de Alimentação do Trabalhador - PAT. Relatório Final sobre o Programa de Alimentação do Trabalhador (PAT). São Paulo; 2013.

[2] Carvalho da Silva A. De Vargas a Itamar: políticas e programas de alimentação e nutrição. Estud. Av. 1995;9(23):87-107.

[3] Mazzon JA. 40 anos do PAT - Programa de Alimentação do Trabalhador: conquistas e desafios da política nutricional com foco em desenvolvimento econômico e social [internet]. São Paulo: Blucher; 2016 [acesso em 10 ago 2017]. Disponível em: http://assertbrasil.com.br

[4] Ministério do Trabalho e Emprego - MTE. Relatório Total PAT [internet]. Brasília; 2008 [acesso em 20 ago 2016]. Disponível em: http://pat.mte.gov.br

[5] Canella DS, Martins APB, Bandoni DH. Iniquidades no acesso aos benefícios alimentação e refeição no Brasil: uma análise da Pesquisa de Orçamentos Familiares 2008-2009. Cad Saúde Pública. 2016;32(3):1-9.

[6] Ministério do Trabalho e Emprego - MTE. PAT Responde [internet]. Brasília; 2014 [acesso em 20 ago 2016]. Disponível em: http://acesso.mte.gov.br

[7] Instituto Brasileiro de Geografia e Estatística - IBGE. Microdados e documentação da POF 2008-2009 [internet]. Rio de Janeiro; 2010 [acesso em 15 out 2016]. Disponível em: ftp://ftp.ibge.gov.br/Orcamentos_Familiares/Pesquisa_de _Orcamentos_Familiares_2008_2009/Microdados

[8] Instituto Brasileiro de Geografia e Estatística - IBGE. Pesquisa de Orçamentos Familiares 2008-2009: despesas, rendimentos e condições de vida [internet]. Rio de Janeiro; 2010 [acesso em 15 out 2016]. Disponível em: http://www.ibge.gov.br/home/estatistica/populacao/condi caodevida/pof/2008_2009/default.shtm.

[9] Hoffmann R. Uso e interpretação das razões de concentração e sua aplicação à análise da insegurança alimentar no Brasil. Segur Aliment Nutr. 2014;21(2):481-498.

[10] Hoffmann R. Condicionantes do sobrepeso e da obesidade de adultos no Brasil, 2008-2009. Segur Aliment Nutr. 2012:19(2):1-16.

[11] Veloso IS, Santana VS, Oliveira NF. Programas de alimentação para o trabalhador e seu impacto sobre ganho de peso e sobrepeso. Rev Saúde Pública. 2007;41(5):769-76. 